\section{Exudative AMD subtypes and eligibility for treatment with ranibizumab}

\begin{abstract}
Purpose To ascertain the proportion of patients with neovascular age-related macular degeneration (AMD) eligible for intravitreal treatment with monoclonal antibodies to vascular endothelial growth factor, on the basis of inclusion criteria used in pivotal clinical trials.

Methods We scrutinised an imaging database
\end{abstract} and extracted all fluorescein angiograms (FAs) captured between 1 January and 31 December 2001. Of the $1083 \mathrm{FA}$, we found 184 where features of AMD in one or both eyes that were observed. In 130 eyes with neovascular AMD, we measured the area of choroidal neovascularisation $(\mathrm{CNV})$ and retinal angiomatous proliferation (RAP). If contiguous to the area of neovascularisation, any areas of blood, exudate, blocked fluorescence, fibrosis, and atrophy were also measured. Descriptive statistics on lesion location, size, and composition were generated and $\chi^{2}$-tests were used to test for associations. Results Of 130 eyes with neovascular AMD, a subfoveal CNV was present in over $75 \%$. Overall, $24.6 \%$ were wholly or predominantly classic, and the remainder minimally classic or occult. Of this latter group, RAPs constituted nearly a third and were significantly associated with co-existent pigment epithelial detachment (PED). Using MARINA and ANCHOR study criteria, less than $50 \%$ would have been eligible for clinical trials for antiangiogenic therapy.

Conclusions The majority of CNV were ineligible for treatment with antiangiogenic therapies when assessed solely on angiographic features. RAPs accounted for some one-third of lesions classified as minimally classic or occult at presentation, and the majority of these would have been
S George ${ }^{1}$, C Cooke $^{1}$ and U Chakravarthy ${ }^{2}$

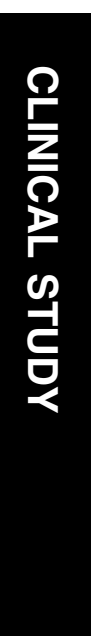

ineligible for inclusion in the pivotal randomized control trials.

Eye (2010) 24, 1247-1251; doi:10.1038/eye.2009.301; published online 18 December 2009

Keywords: age-related macular degeneration; choroidal neovascular membrane; fluorescein angiogram; analysis; anti-VEGF; eligibility

\section{Introduction}

The majority of visual loss from age-related macular degeneration (AMD) is because of neovascularisation involving the central macula. ${ }^{1}$ Currently, argon laser photocoagulation, photodynamic therapy (PDT) with verteporfin and intravitreally delivered antiangiogenic agents are recognised as conferring benefit. $^{2-5}$ Pivotal and post-licensing studies of PDT and antiangiogenic agents have suggested that lesion position and type influences the response to treatment. ${ }^{3,6,7}$ Healthcare providers who are faced with expensive new therapies may seek to limit treatment to specific clinical groups who were shown to experience maximum benefit or to patients who fulfil the eligibility criteria that were set in the key licensing trials. Examples of this include the guidance that has been issued by the National Institute for Clinical Excellence (NICE) in the United Kingdom, limiting PDT treatment to specific angiographic subtypes and more recently by issuing guidance on eligibility for treatment with antiangiogenic agents. ${ }^{8,9}$ Several investigators have previously undertaken exercises to determine the proportion of eligible patients as new therapies have emerged onto the treatment scene. ${ }^{10-13}$ To date, no studies have been undertaken to determine the proportion of patients who would fulfil the
${ }^{1}$ Ophthalmology

Department, Eye and Ear Clinic, Belfast Trust, Royal Group of Hospitals, Belfast, Northern Ireland, UK

${ }^{2}$ Centre for Vision Science, Queen's University Belfast, Belfast, UK

Correspondence: S George, Ophthalmology Department, Eye and Ear Clinic, Belfast Trust, Royal Group of Hospitals, Grosvenor Road, Belfast, UK

Tel: (0) 28 90632392;

Fax: (0) 2890630744

E-mail: sonja_AC@

yahoo.com

Received: 1 July 2009 Accepted in revised form: 30 October 2009 Published online: 18 December 2009 Northern Ireland BT12 6BA 
eligibility criteria used in the key licensing trials, which showed benefit from the use of ranibizumab, a monoclonal antibody inhibitor of vascular endothelial growth factor. We considered it important in the UK setting to describe the characteristics of patients with neovascular AMD to determine the proportions of patients who may be eligible for treatment with ranibizumab.

\section{Materials and methods}

Two of the authors (CC and SG) interrogated an archive of digital photographic and angiographic retinal images stored in an electronic database at the Royal Victoria Hospital, Belfast, which is the tertiary referral centre for the retinal service in Northern Ireland. All imaging entries between 1 January and 31 December 2001 were retrieved using the image net search facility. The following strategy was used to identify all imaging entries with a diagnosis of early or late AMD. An initial scrutiny was performed on any colour images and/or angiograms that fell within the search period. Each set of images (colour, fluorescein, and indocyanine green angiograms, if available) from any one patient was briefly examined to select out those with the features of AMD, and this information was recorded in a customised database. Fundus images and angiograms from patients who were younger than 55 years of age at the date of acquisition were excluded, as our definition of AMD restricted the diagnosis to persons aged 55 years and above. Fundus images and angiograms with features suggestive of high myopia, chronic central serous retinopathy, macular oedema of any other cause, diabetic retinopathy, idiopathic, and/or inflammatory neovascularisation were scrutinised by the senior retinal specialist (UC) before exclusion.

At the next stage, stereoscopic pairs of both colour images and selected early and late fluorescein angiographic frames were retrieved only from the image sets, which had been categorized as AMD at the initial scrutiny. As ICG angiography has never been used in clinical trials to determine eligibility, we did not subject the few ICG angiograms that were also available to systematic analysis. A systematic protocol-based approach was used to record features indicative of the presence of AMD. Colour fundus images were displayed on screen using the IMAGEnet software (TRC 50 IX fundus camera, Topcon Medical Systems Inc., Paramus, NJ, USA). If stereo-pair capture had been used, the pairs of images were examined with stereoviewers. Features that were present in the macular field, which were taken to indicate the presence of neovascular AMD, included retinal elevation due to subretinal fluid, haemorrhage, exudates, pigment epithelial detachment (PED), retinal pigment epithelial tear, and sub or intra-retinal fibrosis. Geographic atrophy was defined as a well-defined area of retinal pigment epithelial atrophy containing visible large choroidal vessels within this area. The presence of any features of early AMD consisting of drusen and/or pigmentary irregularities was also noted.

If an exudative lesion due to AMD was judged to be present on colour images, a full grading of any accompanying fluorescein angiographic run was undertaken. The exudative AMD lesion was defined as all choroidal neovascularisation $(\mathrm{CNV})$ and other lesion components (blood, elevated blocked fluorescence not due to blood, serous PED, fibrosis, and atrophy) contiguous to CNV that could obscure the margins of the $\mathrm{CNV}$ or represent $\mathrm{CNV}$. The area of the entire lesion was outlined on screen and measured along with the greatest linear diameter. Classic and occult CNV and other lesion components were also individually outlined and measured. Any areas of scarring which appeared to be due to thermal laser were delineated separately and not included in lesion composition determination or in lesion area measurements. Data were recorded on a purpose-designed proforma. The image analysis was performed independently by two graders. Any queries or discrepancies in angiographic subtyping were arbitrated by the senior retinal specialist (UC).

In addition, we also examined angiograms captured between 1 March 2004 and 31 March 2004. We chose to examine this second period as a consistency check.

\section{Statistical analysis}

All data were entered into a database and analysed using the statistical package for social sciences, SPSS v12 (SPSS Inc., Chicago, IL, USA). Summary statistics on lesion parameters were generated. Eyes were classified into one of four categories: classic with no occult, predominantly classic, minimally classic, and occult with no classic. A one-way ANOVA was used to compare the area of the lesion by category of classic CNV. The association between lesion components and presence of a retinal angiomatous proliferation (RAP) was examined through cross tabulation and $\chi^{2}$-statistics generated. The data from the second sampling period was not included in the analysis.

\section{Results}

One thousand and eighty three entries were found in the angiographic database in 2001. On the basis of scrutiny of colour and angiographic images, AMD was detected in 184 image sets. Of these, 136 were from female patients (74\%) and the mean age of the 184 AMD cases was 
Table 1 Breakdown of CNV location

\begin{tabular}{lrr}
\hline CNV Location & $\mathrm{n}$ & Percent \\
\hline Subfoveal & 98 & 76.6 \\
Juxtafoveal & 11 & 8.5 \\
Extrafoveal & 10 & 7.7 \\
Peripapillary & 9 & 6.9 \\
Missing data & 2 & 1.5 \\
Total & 130 & 100.0 \\
\hline
\end{tabular}

Table 2 Breakdown of CNV subtype

\begin{tabular}{lcc}
\hline CNV subtype (N) & Percent & Lesion area $\mathrm{mm}^{2}$ (s.d.) \\
\hline Wholly classic (15) & 11.53 & $2.6(2.4)$ \\
Predominantly classic (17) & 13.07 & $6.1(4.4)$ \\
Minimally classic (48) & 36.92 & $9.3(5.9)$ \\
Occult no classic (50) & 38.46 & $11.6(9.3)$ \\
\hline
\end{tabular}

75 years (range 46-94). Neovascular AMD was present in 130 of the $184(71 \%)$ patients.

There were 107 angiograms that were acquired during the second sampling period (March 2004), and late AMD was found in 27 with 14 classified as having exudative features.

More than three-quarters of patients with neovascular AMD had subfoveal involvement (Table 1). Where the lesion did not involve the geometric centre of the fovea, these were split roughly in thirds as juxtafoveal, extrafoveal, and peripapillary. In the breakdown of CNV subtypes (Table 2), wholly or predominantly classic membranes together accounted for less than a quarter of the lesions with minimally classic and occult with no classic CNV accounting for the rest. Neovascular lesion area increased gradually with increasing occult $\mathrm{CNV}$ and this trend was highly statistically significant (ANOVA $P<0.001$ ). The likelihood of exhibiting hard exudates increased with increasing proportion of occult neovascularisation $(P=0.048)$. RAPs constituted $21.7 \%$ of neovascular AMD cases. Of the 28 eyes classified as having RAP, only 4 did not have an associated PED and this was highly statistically significant $\left(\chi^{2}\right.$-test $\left.P<0.001\right)$.

Fewer than half of the angiograms fulfilled the angiographic eligibility criteria of either $\mathrm{ANCHOR}^{3}$ (classic or predominantly classic) or MARINA ${ }^{4}$ (minimally classic and occult with no classic). The reasons for ineligibility for the half that would not have passed ANCHOR and MARINA angiographic criteria are shown in Table 3 . Of the 76 angiograms that fell into the ineligible category, the majority were excluded because the CNV occupied less than $50 \%$ of the total lesion (33\%), with the lesion not involving the foveal centre accounting for a further quarter $(23 \%)$. The vast
Table 3 Ineligible lesions - frequencies and features

\begin{tabular}{lrr}
\hline Ineligibility criteria & $\mathrm{n}$ & Percent \\
\hline Size $>12$ disc areas and or blood $>50 \%$ & 3 & 2.3 \\
Location of CNV (extrafoveal, juxtafoveal, & 30 & 23.1 \\
or peripapillary) & 43 & 33.0 \\
CNV < $50 \%$ of lesion & 43 \\
\hline
\end{tabular}

majority (69 of 76) were ineligible on more than 1 of the exclusion criteria.

Lesions classified as RAP were more likely to be ineligible, as the CNV was usually less than $50 \%$ of the lesion. When compared with eyes with no RAP (23 of 67 ), eyes with RAP (15 of 25 ) were more likely to be ineligible ( $\chi^{2}$-statistic $\left.10.4, P=0.002\right)$.

Grading of fellow eyes showed that two-thirds (64.5\%) had drusen or pigmentary irregularities and just over a quarter $(27.1 \%)$ had a disciform scar. Information on the fellow eye was missing in 23 cases (17.7\%).

\section{Discussion}

In this study, we systematically extracted information from an imaging database with the aim of examining the profile of neovascular AMD cases that would present to a busy tertiary ophthalmology centre in the United Kingdom.

Just under one-half of the angiograms classified as having neovascular AMD in the present study fulfilled the angiographic criteria for selection of patients for entry into the key licensing trials that confirmed benefit with ranibizumab. These criteria are also commonly applied to most other clinical trials that have been undertaken or are currently ongoing in this field. A key reason for ineligibility was the stipulation that the area of neovascularisation should constitute more than $50 \%$ of the lesion. Interestingly, RAP accounted for a large proportion where less than $50 \%$ of the lesion was constituted by CNV. In such eyes, neovascularisation occupied a small area and did not usually involve the geometric centre of the fovea, while the PED (which was most often serous) occupied a large area.

Retinal angiomatous proliferations are recognised as a common feature of AMD, and in terms of their management are known to have a poor visual prognosis. ${ }^{14}$ A spate of small studies have suggested that RAP lesions may be sensitive to VEGF inhibitor therapy and/or combination therapies with PDT and triamcinolone. ${ }^{15-17}$

The next most common reason for ineligibility was the location of the neovascular complex with $23.1 \%$ of scrutinised angiograms showing lesions that did not involve the foveal centre. This definition was less 
stringent than those used in the clinical trials, in which the CNV rather than the lesion is required to involve the foveal centre. Using the more stringent classification, a further two eyes would have been ineligible for inclusion. Our study findings showing that the majority of cases have subfoveal involvement is in keeping with the many observations of many trials which have demonstrated that some three-quarters of eyes at initial presentation have foveal involvement. ${ }^{3-6}$ Our study is also in accordance with previous work showing that the most frequently encountered leakage pattern is occult. 9,10 $^{-10}$

The presence of occult CNV also resulted in much larger lesions. Notably, lesions that contained a higher proportion of occult neovascularisation were on average three times as large in area and twice as great in linear diameter than wholly classic membranes, and a stepwise gradient of increase in area was discernable with increasing proportion of occult. Similar findings have been reported recently in a study by Olsen et al. ${ }^{12}$ In addition, significantly more occult membranes were associated with exudate in agreement with proposals that classic neovascularisation is a more focal disease process than occult. ${ }^{13}$

This study is a retrospective analysis of angiograms within a clinical database and as such has limitations. We did not have visual acuity data and therefore we do not know how many additional persons would have been excluded on this parameter or indeed on other exclusion criteria. We also did not have information on whether any of the patients had received treatment for neovascular AMD. However, we do not believe that the lack of information in this regard is a confounder for several reasons. First, in 2001, thermal laser was the mainstay of treatment, with fewer than $10 \%$ of all patients presenting with neovascular AMD falling into the treatable category, that is, with extrafoveal classic CNV. It was a common practice for patients who received thermal laser to be reviewed for a very short period with the vast majority discharged when visual outcomes indicated the futility of continuing treatment. Therefore, we believe that very few of the angiograms would have come from patients receiving thermal laser. Furthermore, as thermal laser leaves a distinctive scar, this area would have been excluded in the determination of lesion size and characteristics and thus would not have confounded measurements or lesion subtype classification. PDT which extended the treatable percentages and which resulted in large numbers of patients returning for follow-up was only introduced into the United Kingdom towards the latter half of 2003 and into Northern Ireland in the summer of 2004, which is after we had completed our sampling.

Our analyses show that less than half of the patients detected with exudative AMD would have passed the angiographic eligibility criteria used in the pivotal clinical trials on anti-VEGF therapies. Use of stringent inclusion and exclusion criteria often results in the exclusion of the majority of the population with the disorder. When technologies are introduced into clinical practice and when all patients with the disorder are treated, the results may or may not reflect those observed in the controlled clinical trials.

\section{Summary}

What was known before

- Pivotal studies of antiangiogenic agents have suggested that $\mathrm{CNV}$ position and type influences treatment response. Several investigators have previously undertaken exercises to determine the proportion of eligible patients as new therapies have emerged.

What this study adds

- No studies have been undertaken to determine the proportion of patients who fulfil the eligibility criteria used in key trials demonstrating benefit from ranibizumab; we considered this important in the UK setting. The majority of $\mathrm{CNV}$ were ineligible for treatment with antiangiogenic therapies when assessed solely on angiographic features. RAPs accounted for one-third of minimally classic or occult lesions and the majority were ineligible for inclusion in the pivotal randomized control trials.

\section{Conflict of interest}

The authors declare no conflict of interest.

\section{Acknowledgements}

We thank the Fluorescein Angiography and Ophthalmic Photography Department at the Royal Victoria Hospital, Belfast.

\section{References}

1 Wong TY, Chakravarthy U, Klein R, Mitchell P, Zlateva G, Buggage $\mathrm{R}$ et al. The natural history and prognosis of age-related macular degeneration: a systematic review of the literature and meta-analysis. Ophthalmology 2008; 115: 116-126.

2 Chakravarthy U, Soubrane G, Gandello F, Chong V, Creuzot-Garcher C, Dimitrakos SA et al. Evolving European Guidance on the medical management of neovascular age-related macular degeneration. Br J Ophthalmol 2006; 90: 1188-1196.

3 Photodynamic Therapy of Subfoveal Choroidal Neovascularisationin Age-Related Macular Degeneration With Verteporfin. One-Year Results of 2 Randomised Clinical Trials TAP - Report 1. Arch Ophthalmol 1999; 117: 1329-1345.

4 Rosenfeld PJ, Brown DM, Heier JS, Boyer DS, Kaiser PK, Chung CY et al. Ranibizumab for neovascular age-related macular degeneration. N Engl J Med 2006; 355(14): 1419-1431. 
5 Brown DM, Kaiser PK, Michels M, Soubrane G, Heier JS, Kim RY et al. Ranibizumab versus verteporfin for neovascular age-related macular degeneration. $N$ Engl J Med 2006; 355(14): 1432-1444.

6 Verteporfin in Photodynamic (VIP) Therapy Group. Verteporfin Therapy of Subfoveal Choroidal Neovascularization in Age-Related Macular Degeneration - Two year Results of a Randomised Clinical Trial Including Lesions With Occult With No Classic Choroidal Neovascularistion - Verteporfin in Photodynamic Therapy Report 2. Am J Ophthalmol 2001; 131: 541-560.

7 Lee AY, Raya AK, Kymes SM, Shiels A, Brantley Jr MA. Pharmacogenetics of complement factor $\mathrm{H}(\mathrm{Y} 402 \mathrm{H})$ treatment of exudative age-related macular degeneration with ranibizumab. Br J Ophthalmol 2009; 93: 610-613.

8 NICE. Macular degeneration (age-related) - photodynamic therapy. The Clinical effectiveness and cost effectiveness of photodynamic therapy for age-related macular degenration. http://guidance.nice.org.uk/TA68.

9 NICE. Macular degeneration (age-related) - ranibizumab and pegaptanib. http://guidance.nice.org.uk/TA155.

10 Margherio RR, Margherio AR, DeSantis ME. Laser treatments with vertepofin therapy and its potential impact on retinal practices. Retina 2000; 20: 325-330.

11 Haddad WM, Coscas G, Soubrane G. Eligibility for treatment and angiographic factors at the early stage of exudative age related macular degeneration. Br J Ophthalmol 2002; 86: 663-669.

12 Olsen TW, Feng X, Kasper TJ, Rath PP, Steuer ER. Fluorescein angiographic lesion type frequency in neovascular age-related macular degeneration. Ophthalmology 2004; 111(2): 250-255.

13 Cohen SY, Creuzot-Garcher C, Darmon J, Desmettre T, Korobelnik JF, Levrat $\mathrm{F}$ et al. Types of choroidal neovascularisation $(\mathrm{CNV})$ in newly diagnosed exudative age-related macular degeneration. Br J Ophthalmol 2007; 91: 1173-1176.

14 Viola F, Massacesi A, Orzalesi N, Ratiglia R, Staurenghi G. Retinal angiomatous proliferation: natural history and progression of visual loss. Retina 2009; 29: 732-739.

15 Gharbiya M, Allievi F, Recupero V, Martini D, Mazzeo L, Gabrielli CB. Intravitreal bevacizumab as primary treatment for retinal angiomatous proliferation: twelve-monht results. Retina 2009; 29: 740-749.

16 Konstantinidis L, Mameletzi E, Mantel I, Pouranaras JA, Zografos L, Ambresin A. Intravitreal ranibizumab (Lucentis(R) in the treatment of retinal angiomatous proliferation (RAP). Graefes Arch Clin Exp Ophthalmol 2009; 247(9): 1165-1171.

17 Rouvas AA, Papakostas TD, Vavvas D, Vergados I, Moschos $\mathrm{MM}$, Kotsolis A et al. Intravtireal ranibizumab, intravitreal ranibizumab with PDT, and intravitreal triamcinolone with PDT for the treatment of retinal angiomatous proliferation: a prospective study. Retina 2009; 29: 536-544. 